\title{
Un diálogo entre música y ciencias exactas a partir de una correspondencia metafórico-analógica con el concepto de "estructuras disipativas" de Ilya Prigogine (Phowa para flauta bajo sola, un caso de estudio)
}

Pablo Federico Araya

pf.araya79@gmail.com

Recepción: marzo 2020.

Aceptación: junio 2020.

\section{Resumen}

El trabajo intenta profundizar sobre la relación entre música y ciencias exactas a partir de la mirada de Ilya Prigogine. En tal sentido, primero se realiza un breve recorrido histórico que explica cómo fue dándose la vinculación entre ambos campos; seguidamente se busca entender los límites y posibilidades de dicha vinculación apelando a una revisión crítica del asunto; posteriormente se observa que una de las vías factibles para este diálogo es a través de la metáfora y la analogía: lo uno y lo otro fungen como herramientas concretas al momento de establecer el vínculo entre música y ciencias exactas; por tal motivo, desarrollar lo metafórico y lo analógico resulta indispensable. Finalmente se explicita el enfoque de metaforizaciónanalogización en sí, o sea, se toma la obra Phowa (2018) para flauta bajo sola y se muestra que esta posee una semejanza estructural con la noción de "estructuras disipativas" desarrollada por Prigogine. Ahora bien, el cruce de dominios al que se ha recurrido (metáfora/analogía), significa que, en la actualidad, se han puesto de manifiesto "nuevos" mecanismos creativocognitivos al momento de imaginar y pensar la música (particularmente aquella que utiliza sonoridades tímbricas muy complejas).

Palabras clave: música, ciencias exactas, metáfora, analogía, estructuras disipativas
A Dialogue between Music and Exact Sciences from a Metaphorical-Analogical Correspondence with the Concept of "Dissipative Structures" of Ilya Prigogine (Phowa for solo bass flute, a case study) 


\begin{abstract}
The work intends to delve into the relationship between music and exact sciences form Ilya Prigogine's point of view. So, first there is a brief historical tour that shows how the bonding of these two fields occurred; second, we try to understand which the limits of this bonding are; then, it is observed that one of the feasible ways for this dialogue to take place is through metaphor and analogy: both function as concrete tools when establishing the link between music and exact sciences; that is why understanding and developing the concepts of metaphor and analogy is crucial. Finally, the metaphorization-analogization approach itself is explained, namely, the piece for solo bass flute called Phowa (2018) is taken as an example, and it is shown that it has a structural similarity with the notion of "dissipative structures" developed by Prigogine. Nevertheless, the crossing domain (metaphor/analogy) means that, at present, "new" cognitive-creative mechanisms have been developed by the composers at the moment of imagining and thinking their music (in particular, those musics that use very complex sonorities).
\end{abstract}

Keywords: Music, exact sciences, metaphor, analogy, dissipative structures

\title{
Um diálogo entre música e ciências exatas a partir de uma correspondência metafórico-analógica com o conceito de "estruturas dissipativas" de Ilya Prigogine (Phowa para flauta baixo solo, um caso de estudo)
}

\section{Resumo}

O trabalho procura aprofundar a relação entre música e ciências exatas na perspectiva de Ilya Prigogine. Nesse sentido, primeiramente se realiza um breve percurso histórico que explica como começou a ligação entre os dois campos; em seguida, procura-se entender os limites e as possibilidades dessa ligação através de uma revisão crítica do assunto; posteriormente, observa-se que uma das vías possíveis para esse diálogo pode ser feita por meio da metáfora e da analogia: uma e outra funcionam como ferramentas concretas no momento de se estabelecer o vínculo entre música e ciências exatas; por esse motivo, é essencial desenvolver o metafórico e o analógico. Finalmente, explicita-se o enfoque da metaforação-analogização em si, isto é, toma-se a obra Phowa (2018) para flauta baixo solo, e mostra-se que esta possui uma semelhança estrutural com a noção de "estruturas dissipativas" desenvolvidas por Prigogine. Contudo, o cruzamento de domínios que foram utilizados (metáfora/analogía), significa que "novos" mecanismos 
criativos-cognitivos foram revelados no momento de imaginar e pensar a música (particularmente aquele que utiliza sonoridades tímbricas muito complexas).

Palavras-chave: música, ciências exatas, metáfora, analogia, estruturas dissipativas

\section{Introducción}

El presente escrito intenta aportar al diálogo entre ciencias exactas y humanidades, denominadas por el Nobel en química Ilya Prigogine (1997) como "dos culturas" ${ }^{1}$ (aunque los posibles aportes que aquí se generen procuran echar luz sobre cuestiones creativo-musicales solamente). Pero, para que el intercambio y la comunicación entre ambas culturas sea efectivo, o mejor, para que las experiencias e ideas de una disciplina como la científica sean aprovechadas al máximo por lo artístico-musical, aquí propondremos un horizonte teórico que explica que la apropiación de algunas ideas provenientes de la matemática y la física actuales (no-linealidad, inestabilidad, turbulencia, etc.), serán sumamente útiles para entender y explicar lo que acontece en algunas obras instrumentales (solistas y de cámara) de factura reciente (podrían incluirse obras de diferentes autores tales como Julio Estrada, Horacio Vaggione, Marcos Franciosi, Juan C. Tolosa, Natalia Solomonoff, Gerald Eckert, Luciano Azzigotti, etc.; no obstante, como caso de estudio y observación se tomará a Phowa ${ }^{2}-2018-$, composición para flauta bajo sola de quien escribe este artículo).

\section{Las dos culturas: una breve aproximación histórica}

La relación entre música y ciencia, al menos en Occidente, se manifiesta desde hace mucho tiempo: por ejemplo, en la antigua Grecia fue Pitágoras (filósofo y matemático) quien estudió las proporciones de los intervalos

\footnotetext{
1 "Resulta evidente que este nuevo enfoque aúna ciencias y humanidades. Tradicionalmente, las ciencias se ocupaban de universales, y las humanidades de acontecimientos. Actualmente, es la interpretación humanista de la naturaleza en términos de acontecimientos lo que se difunde en la propia ciencia. Por lo tanto, no es sorprendente que algunos conceptos que recientemente hayan sido puestos de relieve encuentren explicación simultáneamente en las ciencias y en las humanidades" (Prigogine, 1997, p. 176)

${ }^{2}$ Obra estrenada por Beatrix Wagner en el Move around concert el 17 de agosto de 2018 en Eckernförde, (Alemania) y el 17 de octubre de 2019 en las "Micro-Jornadas de Composición y Música Contemporánea" en Córdoba (Argentina). Recuperado de https://www.youtube.com/watch?v=0DX2omz dDc (concierto en Alemania) / https://www.youtube.com/watch?v=XxqQjquhdFU (concierto en Argentina).
} 
musicales (Amster, 2004); también en la Edad Media existió, en la enseñanza de las escuelas carolingias, lo que se llamaba el quadrivium (cuatro vías) científico: cuestión que comprendía y articulaba a la aritmética, la geometría, la astronomía y la música (Hoppin, 1991). Un poco más avanzado en el tiempo, y basándose en la perspectiva del mismo Pitágoras y de Platón, tanto Johannes Kepler (1571-1630) como Marin Mersenne (1588-1648) sostuvieron que la estructura del universo guardaba una estrecha similitud con la "armonía musical"; sin embargo, este parecido con lo musical podía ser expresado y entendido en términos matemáticos (Wollenberg, 2006). En sintonía con lo recién expresado, aunque ya en el siglo XXI, el físico teórico Brian Greene (científico vinculado a la teoría de cuerdas) explicaba que el universo no está constituido por partículas elementales puntuales, sino por "estados vibracionales" llamados cuerdas, lo cual supone una suerte de "reinterpretación" de los enfoques ya trazados por Pitágoras, Platón o Kepler en relación con el cosmos y su vinculación con lo musical (Amster, 2019).

También, pero ahora desde el lado de la música, el compositor inglés William Crotch (1775-1848) postuló que la música debía comprenderse como una actividad "artística y científica" a la vez (Wollenberg, 2006). En la misma Iannis Xenakis (1922-2001) utilizó herramientas y modelos matemáticos para formalizar su música (Xenakis, 1971). Igualmente, pero sin echar mano de manera directa o explícita a procedimientos o formalismos matemáticos en lo que a composición musical se refiere, tanto Julio Estrada (1943-) como Horacio Vaggione (1943-) han tenido una vinculación muy próxima con ideas o conceptos provenientes del campo científico, entre otros: las nociones de no-linealidad, de multidimensionalidad, de multiescala, etc. (Budón, 2000; Estrada, 2002).

Como vimos, y si continuáramos indagando respecto a los lazos de proximidad entre música y ciencia a lo largo de la historia, se concluiría que esta relación fue mucho más fecunda de lo que se piensa, solo que, a partir del siglo XVII, la música empezó a distanciarse de las "ciencias exactas" para empezar a formar parte de las "bellas artes" (Fauvel, Flood y Wilson, 2006). No obstante, y como algunos teóricos del arte han señalado, la vinculación y la incorporación de la música al ámbito de las "bellas artes" no estuvo ni está exenta de tensiones. Es decir, a veces no está muy claro hasta qué punto la música sigue dentro de los límites del "arte":

Mitchell explica el estatus ajeno de la música en relación con las otras artes de dos maneras: la música ha renunciado al "territorio" disputado por la poesía o la pintura debido a la ausencia de "referencia, 
de representación, de denotación y de significado"; y también, la exclusión de la música en esta conversación con las demás artes, ha derivado en una "guerra de signos" que ha sido interpretada como una oposición binaria básica entre la imagen y la palabra [...] Asimismo, él retiene a la música como un extraño, lo que hace que uno se pregunte por qué menciona a la música después de todo. Aun así, les recuerda a sus lectores que, aunque la música ha sido excluida, "todas las artes" se han mantenido durante mucho tiempo "procurando aspirar a la condición de la música" (Goehr, 2017, pp. 140-141). ${ }^{3}$

\section{Las dos culturas: una revisión crítica del asunto}

Lo esbozado arriba demuestra, como ya se dijo, que música y ciencia poseen una cercanía bastante singular. Asimismo, queda claro que ambas disciplinas no son lo mismo. Por consiguiente, primero tengamos una aproximación respecto al trabajo científico (aunque sea breve), y posteriormente intentemos comprender hasta qué punto puede vincularse con lo artístico con lo musical.

El físico y matemático Eugene Wigner (1902-1995) señalaba que uno de los propósitos más importantes de la física es el de advertir ciertas regularidades en los fenómenos naturales (y algo similar decían otros autores respecto a la matemática: "En el corazón de las matemáticas está la búsqueda de las regularidades, las estructuras, los patrones” Nickerson, 2011, p. 3). ${ }^{4}$ Por lo tanto, si estas regularidades se confirman indepen-

\footnotetext{
3 "Mitchell explains music's outsider status in two ways: that music has renounced the contested 'territory' of poetry and painting, of 'reference, representation, denotation, and meaning', and that music's exclusion from conversation has suited a 'war of signs' construed according to a basic binary opposition between word and image [...] He retains music as an outsider, prompting one to wonder why he mentions music at all. Still, he does remind to his readers, although music has been excluded, 'all the arts' have long been held 'to aspire to the condition of music'”' (Goehr 2017, pp. 140-141).

4 "At the heart of mathematics is the search for regularity, for structure, for pattern [...]" (Nickerson, 2011, p. 3). Asimismo, cuando se dice que en la matemática se encuentran regularidades, al igual que en el ámbito de la física, esto se refiere, en líneas generales, al interrogante que proponen Wigner (2004) y muchos otros autores (Hacking, 2014; Gelfert, 2016): ¿cómo es posible que la matemática sea tan eficaz para describir los diversos fenómenos de la naturaleza que se estudian desde la física? 0 también ¿A qué se debe que un lenguaje tan riguroso y estructurado como el de la matemática sirva para poder explicar y describir -en términos físicos-a fenómenos naturales tan variados? Es decir ¿cuál es la conexión entre los modelos matemáticos -que muchas veces expresan regularidades, patrones y estructuras-y los fenómenos físicos? Algunos de los especialistas que recién citamos dicen que los modelos matemáticos son "analogías" o "metáforas" que representan, no sin ciertas limitaciones, a los fenómenos físicos. En definitiva, podría decirse que lo que está en discusión en este punto, tiene que ver con el hecho de preguntarse si la naturaleza -que es estudiada, entre otras áreas científicas, por la física- realmente responde y se ajusta a esas regularidades o estructuras y búsquedas de patrones que la matemática desarrolla para sí (Wilber, 2007; Nickerson, 2011; Weisberg, 2013; Hacking, 2014; Gelfert, 2016).
} 
dientemente de si suceden en la Tierra o en Neptuno, o de si suceden a un nivel escalar minúsculo o mayúsculo, pues entonces se constituyen como "leyes generales de la naturaleza" (Wigner, 2004). Desde luego, Wigner también aclara que existen limitaciones en el establecimiento de tales "leyes" ya que solo representan un conocimiento acotado de una porción de la realidad. Ahora bien, las "leyes de la naturaleza" se expresan con un lenguaje matemático. Esto significa que las matemáticas son el lenguaje con el que los físicos describen las "regularidades" que observan en los fenómenos naturales. En consecuencia, las matemáticas son una herramienta fundamental que impiden que la formulación de tales "leyes" sea imprecisa y contradictoria a la vez.

De todas maneras, la descripción que Wigner nos ha ofrecido en relación con la ciencia no es definitiva ni concluyente: científicos como Ilya Prigogine cuestionaron fuertemente esta pretensión de universalizar y matematizar todo. ${ }^{5}$ Sin embargo, y más allá de la crítica que Prigogine u otros la hayan llevado a cabo, lo cierto es que música y ciencia, a pesar de algunos de sus parecidos, poseen finalidades y propósitos muy distintos: por ejemplo, y resulta obvio, la música no tiene como fin buscar ni encontrar leyes generales que posteriormente deben verificarse experimentalmente. No obstante, esto no significa que, en más de una ocasión, tanto lo artístico como lo científico no hayan dialogado, por decirlo de alguna manera, para ampliar sus horizontes o límites. 0 si se quiere, podría decirse que tanto lo artístico como lo científico han conversado para encontrar nuevas formas de responder o encarar a los interrogantes e inquietudes propias (casos emblemáticos que vimos del lado de la ciencia han sido los de Kepler, Mersenne o Greene, y de Xenakis, Estrada o Vaggione del lado de la música). Por ende, si bien reconocemos que el diálogo entre música y ciencias exactas (física y matemática) es sumamente enriquecedor e importante, además de que debe darse sin prejuicios y lo más fluidamente posible, no obstante, creemos que tal diálogo debe llevarse a cabo cuidadosamente.

\footnotetext{
${ }^{5}$ En sus propias palabras: "[...] pensamos que la ciencia de hoy escapa al mito newtoniano porque ha concluido teóricamente en la imposibilidad de reducir la naturaleza a la escondida simplicidad de una realidad regida por leyes universales. La ciencia de hoy no puede ya adjudicarse el derecho a negar la pertinencia y el interés de otros puntos de vista, de negarse en particular a escuchar los de las ciencias humanas, de la filosofía y el arte" (Prigogine y Stengers, 1990, p. 84). Y agrega el Nobel: “Cierto que la matematización constituyó [...] el 'lecho de Procusto' en el que la naturaleza fue simplificada" (Prigogine, 1997, p. 83).
} 


\section{Las dos culturas: lo metafórico y lo analógico como alternativas para el diálogo entre música y ciencias exactas}

La perspectiva de diálogo entre música y ciencias exactas que se propone en este escrito se relaciona con los conceptos de metáfora y analogía. Pero antes de explicitar la metafórización-analogización en sí (es decir, el enfoque específico de conexión y relación entre música y ciencias exactas que aquí desarrollaremos), resultará conveniente tener una aproximación a las nociones de metáfora y analogía.

\subsection{Metáfora}

Casi todos los autores clásicos coinciden en circunscribir la metáfora dentro del ámbito exclusivo de lo literario (con mayor énfasis en la poesía; cuestión que le valió cierto menosprecio o subestimación) (Lakoff, 1993; Visokolskis, 2006). Podría decirse que a la metáfora se la consideró como un recurso artificioso del lenguaje que implicaba una sobrecarga o exageración en el significado de las palabras (Pérez Bernal, 2007). Por lo tanto, la metáfora, en su acepción tradicional vinculada a la poesía, más que como un instrumento cognitivo válido y esclarecedor al momento de establecer los significados y las referencias, en realidad fue percibida como un artilugio lingüístico sin mayor relevancia y con una funcionalidad netamente artístico-expresiva. Sin embargo, estas posturas reduccionistas fueron perdiendo gravitación y poco a poco se empezó a tomar conciencia de las grandes implicancias cognoscitivas que la metáfora tenía. Tanto fue así que estas comenzaron a ganar terreno infiltrándose en diversos ámbitos y actividades del conocimiento humano (por ejemplo, la ciencia). Precisamente, una de las personalidades que se encargó de otorgarle un sentido diferente a lo metafórico fue el lingüista norteamericano George Lakoff. Según su teoría, la metáfora no debe comprenderse como una mera novedad del lenguaje. Por el contrario, esta debe entenderse como un elemento y un atributo fundamental del pensamiento. 0 mejor aún, la metáfora es una herramienta o instrumento del pensamiento que ayuda a conceptualizar y estructurar la realidad cotidiana a través del lenguaje y de la confección, y cruce de mapeos mentales que pertenecen a dominios existenciales distintos (conceptualizaciones y mapeos que además tienen una incidencia decisiva en la forma en que las personas experimentan y vivencian el mundo que les rodea e incluye) (Lakoff y Johnson, 1986; Lakoff, 1993). Así pues, desde la perspectiva recién mencionada, se llega a la conclusión de que las metáforas no son más que mapeos conceptuales de ámbitos cognitivos y experienciales diferentes que, por medio de la facultad del pensamiento, se conectan y corresponden entre sí. En consecuencia, y reiteramos enfáticamente, es el pensamiento el que advierte e instituye la correspondencia ontológica de los mapeos que se 
conectan y relacionan, aunque, a posteriori y consecuentemente con ello, será el lenguaje (en principio) el que buscará una vía adecuada para expresar el cruce y correspondencia de los mapeos:

La metáfora implica entender un dominio de la experiencia [...] en términos de otro dominio que es diferente [...] Técnicamente hablando, la metáfora puede ser entendida como un mapeo [...] que va desde un dominio-fuente [...] a otro dominio-objetivo. El mapeo está fuertemente estructurado. Hay correspondencias ontológicas en las que las entidades del dominio[-fuente] [...] se corresponden sistemáticamente con las entidades del dominio[-objetivo] (Lakoff, 1993, pp. 206-207). ${ }^{6}$

Lo que se acaba de explicar deja claro que la teoría del norteamericano posee un enfoque más enraizado en lo cognitivo que en lo lingüístico.

La metáfora no es sólo una cuestión del lenguaje, sino que es algo referido al pensamiento y la razón. El lenguaje es secundario. El mapeo es lo primordial [...] Así pues, como el mapeo [...] es lo primero, entonces esto es lo que ocupa nuestro mayor interés. Por ello, hemos reservado el término "metáfora" para los mapeos conceptuales más que para las expresiones lingüísticas (Lakoff, 1993, pp. 208-209). ${ }^{7}$

\subsection{Analogía}

La analogía o el razonamiento analógico, en su acepción más típica o clásica, implica contemplar las semejanzas existentes entre dos entidades o dominios de la realidad que son diferentes (perspectiva similar a la de Lakoff en relación con la metáfora). Podría argüirse que casi todos los especialistas en el tema, en una primera instancia, coinciden con esta mirada.

Una analogía es una comparación entre dos objetos, o dos sistemas de objetos, que destaca los aspectos que se piensan son similares entre ambos. Asimismo, el razonamiento analógico es cualquier tipo

\footnotetext{
6 "The metaphor involves understanding one domain of experience [...] in terms of a very different domain of experience [...] More technically, the metaphor can be understood as a mapping [...] from a source domain [...] to a target domain. The mapping is tightly structured. There are ontological correspondences, according to which entities in the domain of love [source domain] [...] correspond systematically to entities of the [target] domain (Lakoff, 1993, pp. 206-207).

7 "The metaphor is not just a matter of language, but of thought and reason. The language is secondary. The mapping is primary [...] Since it is the mappings are primary and that state the generalizations that our principal concern, we have reserved the term 'metaphor' for the mappings, rather than for the linguistic expressions" (Lakoff, 1993, pp. 208-209).
} 
de pensamiento que se basa en una analogía [...] El razonamiento analógico resulta fundamental para el pensamiento de los seres humanos [...] el razonamiento analógico ha jugado un importante rol en una amplia gama y variedad de problemas en diferentes contextos. El uso explícito de los argumentos analógicos, desde la antigüedad hasta hoy, ha sido una característica distintiva del razonamiento científico, filosófico y legal (Bartha, 2013). ${ }^{8}$

Igualmente, Macagno, Walton y Tindale señalan lo siguiente:

El razonamiento analógico es un proceso complejo basado en una comparación entre dos o dos pares de entidades o estados de cosas [...] que comparten algunas características comunes [...] Esta comparación es el fundamento de un tipo específico de inferencia (el llamado argumento por analogía) en el que la conclusión consiste en la atribución de una característica específica (una cualidad, un predicado) que caracteriza al análogo con el objetivo (2016, p. 2). ${ }^{9}$

Asimismo, Paul Bartha (2013) sugiere que la analogía posee cuatro roles importantes:

» Un rol heurístico: como un instrumento o herramienta de ayuda para el descubrimiento de posibles soluciones en diversas áreas o disciplinas.

» Un rol de justificación: se da cuando un argumento o un razonamiento analógico actúa como soporte en la construcción de ciertas hipótesis.

» Un rol programático: cuando mediante el uso de la analogía se establece el curso o dirección de un programa investigativo.

» Un rol cognitivo-pedagógico: que busca entender cómo es que los humanos aprendemos apelando al uso de las analogías.

\footnotetext{
8 "An analogy is a comparison between two objects, or systems of objects, that highlights respects in which they are thought to be similar. Analogical reasoning is any type of thinking that relies upon an analogy [...] Analogical reasoning is fundamental for human thought [...] analogical reasoning has played an important, but sometimes mysterious, role in a wide range of problem-solving contexts. The explicit use of analogical arguments, since antiquity, has been a distinctive feature of scientific, philosophical and legal reasoning" (Bartha, 2013).

9 "Analogical reasoning is a complex process based on a comparison between two or two pairs of entities or states of affairs [...] sharing some common features [...] This comparison is the ground of a specific type of inference (the so-called argument from analogy), in which the conclusion consists in the attribution of a specific feature (a quality; a predicate) characterizing the Analogue to the Target [...]" (Macagno, Walton y Tindale, 2016, p. 2).
} 
De todas formas, es importante señalar que la analogía no fue muy bien vista por la lógica tradicional. Esto quiere decir que lo analógico no se consideraba como un argumento lógico válido (por no ser un argumento deductivo). En cambio, la analogía fue percibida como un argumento débil (que formaba parte del discurso cotidiano) cuya finalidad era la persuasión (Palau, 2003; Walton y Hyra, 2018). Sin embargo, desde hace ya algún tiempo, esta perspectiva negativa (ya que como se dijo lo analógico era tomado como una falacia $)^{10}$ tuvo un giro significativo dando lugar a lo que hoy se conoce como "lógica informal" o "teoría de la argumentación o de pensamiento crítico" (perspectivas que tienen un impacto directo e importante en el campo de la filosofía, la ciencia y lo legal). En definitiva, podría decirse que lo analógico presenta una naturaleza dual o ambigua: no posee la contundencia que sí tiene, por ejemplo, el argumento deductivo, no obstante, tampoco deja de otorgar cierta plausibilidad o fortaleza al argumento. En tal sentido, obsérvese lo que Walton (2006) señala:

Esta forma de argumentación es intrínsecamente anulable porque en cualquiera de los dos casos habrá similitudes en algunas cosas, pero también habrá diferencias en otras. Mientras que un caso puede ser, en líneas generales, similar al otro, esto no significa que los dos casos serán iguales en todos sus atributos. Si fueran iguales en todo, entonces se trataría de lo mismo [...] Si un caso es similar al otro en algún respecto, entonces esta similitud otorga un cierto peso y grado de plausibilidad al argumento. Pero, si los dos casos son diferentes en otros aspectos, entonces esa diferencia tiende a socavar el peso de la plausibilidad de dicho argumento. Por ende, los argumentos por analogía pueden ser fuertes o débiles (pp. 96-99). ${ }^{11}$

\section{3. ¿Por qué el uso de la metáfora y la analogía?}

Dijimos que nuestra perspectiva de diálogo entre las dos culturas contiene lo metafórico y lo analógico. Pero ¿a qué se debe esto? Desde la mirada

\footnotetext{
10 "Los textos de lógica del siglo XX tienden a ver a los argumentos por analogía como peligrosos y engañosos. Incluso algunos la clasifican como una falacia basándose en la aceptación de que, en términos generales, los argumentos por analogía son formas falaces de argumentación" (Walton, Reed y Macagno, 2008, pp. 48-49).

11 "This form of argument is defeasible, because any two cases will be similar to each other in certain respects, but dissimilar to each other in other respects. So while one case may be generally similar to another, that does not mean that the two cases will be similar in every respect. If they were similar in every respect, they would be the same case [...] If one case is similar to another in a certain respect, then that similarity gives a certain weight of plausibility to the argument from analogy. But if the two cases are dissimilar in some other respect, citing this difference tends to undermine the plausibility of the argument. So arguments from analogy can be stronger or weaker [...]" (Walton, 2006, pp. 96-99).
} 
de este escrito, ciencias exactas y música son áreas que poseen muchos elementos comunes; enumeramos algunos:

» ambas áreas son intrínsecamente creativas;

» ambas hacen uso de sistemas simbólicos externos (notación específica) que facilitan los procesos creativo-constructivos (Nickerson, 2011; Dutilh Novaes, 2013; Araya, 2019b);

» tanto la música como las ciencias exactas, y especialmente la matemática, deben lidiar con la noción de estructura (Bohm, 2002; Beran, 2004; Hacking, 2014; Araya, 2019b).

Así pues, los elementos en común que acabamos de mostrar entre ambos dominios, habilitan y fomentan al establecimiento de conexiones metafórico-analógicas diversas. No obstante, existe otro aspecto muy destacado en relación a lo metafórico y lo analógico: son dispositivos que ayudan a conocer o esclarecer fenómenos o porciones de la realidad que se entienden poco y que no se sabe con precisión cómo es que funcionan:

El entendimiento de Duhem respecto a la analogía es más específico [...] las analogías consisten en juntar o acercar dos sistemas abstractos, en el que uno que ya es bien conocido, nos sirve y ayuda para entender $\mathrm{y}$ formular mejor al otro que es poco conocido. En esto no hay nada que pueda sorprender o molestar a los lógicos más rigurosos [...] Consideremos el siguiente ejemplo: cuando Christiaan Hyugens (1629-1695) propuso su teoría de la luz, lo hizo en base a una analogía con la teoría ondulatoria del sonido: las relaciones entre los varios atributos y características de la luz, son similares a aquellos formulados en la teoría acústica [...] Entendido así, la analogía se vuelve un instrumento legítimo para conocer y explorar aspectos de un dominio sobre la base de otro (Gelfert, 2016, p. 6). ${ }^{12}$

En consecuencia, y como se verá en la sección próxima, la metáfora-analogía que desarrollaremos con lo que Prigogine denominaba "estructuras

\footnotetext{
12 “Duhem's understanding of 'analogy' is more specific [...] Analogies consist in bringing together two abstract systems; either one of them already known serves to help us guess the form of the other not yet known, or both being formulated, they clarify the other. There is nothing here that can astonish the most rigorous logician [...] Consider the following example: When Christiaan Huygens (1629-1695) proposed his theory of light, he did so on the basis of analogy with the theory of sound waves: the relations between the various attributes and characteristics of light are similar to those described by acoustic theory [...] Thus understood, analogy becomes a legitimate instrument for learning about one domain on the basis of what we know about another" (Gelfert 2016, p. 6).
} 
disipativas", nos ayudará a comprender y definir mejor la estructura y el comportamiento de algunas obras instrumentales (solistas y de cámara) actuales que utilizan una notación estratificada o multidimensional.

\section{Las dos culturas: nuestro enfoque metafórico-analógico}

Como ya fue expresado, el enfoque y proceso de metaforización-analogización que aquí proponemos se vincula con lo que Prigogine ha llamado "estructuras disipativas". Pero ¿qué son las estructuras disipativas? Podría decirse que esta terminología adoptada por el Nobel en química se relaciona con una temática científica que ha estado muy en boga en las últimas décadas, a saber: el problema de la "complejidad" o de los "sistemas complejos". Ahora bien, la idea de complejidad (en el ámbito de las ciencias exactas) necesariamente comprende e incluye otros factores tales como el "caos", la "no-linealidad", la "inestabilidad dinámica”, la "irreversibilidad", la "entropía”, etc. Asimismo, todos estos conceptos que recién nombramos se encuentran inextricablemente unidos. Por lo tanto, el estudio de la complejidad o de los sistemas complejos no resulta ser una tarea sencilla. De todos modos, observemos lo que Prigogine decía respecto a las "estructuras disipativas":

La segunda ley de la termodinámica se ha asociado por antonomasia a la "destrucción" de estructuras [...] El razonamiento más reciente tiene su punto de partida en el hecho de que, en condiciones muy inestables, incluso en el marco de la segunda ley de la termodinámica, pueden surgir nuevas estructuras. Estas nuevas estructuras dinámicas son las "estructuras disipativas" [...] La superposición de un gran número de estas escalas espacio-temporales puede volver a adoptar el aspecto de turbulencia o caos [...] Diremos que los últimos hallazgos sobre situaciones no-lineales muy inestables nos facilitan un magnífico ejemplo sobre la transición de desorden térmico a estructuras macroscópicas, ya sea «orden» o «caos» macroscópico [...] Las estructuras disipativas son consecuencia de procesos irreversibles (1997, pp. 158-161).

Entonces, los componentes básicos de las estructuras disipativas son: la inestabilidad dinámica, la turbulencia, los comportamientos no-lineales, una gran cantidad de componentes funcionando a diferentes escalas espacio-temporales $y$, derivado de lo anterior, a veces puede observarse caos ${ }^{13}$

\footnotetext{
${ }^{13}$ Resulta importante y oportuno señalar lo siguiente: en el caso de autores como Prigogine, la idea
} 
(con el agregado particular de que todo esto puede generar el nacimiento de estructuras novedosas).

Pues bien, ahora que ya disponemos de una descripción más acabada en relación con las estructuras disipativas, veamos cómo es que estas se plasman, en términos metafórico-analógicos, en el plano musical.

\subsection{Primer enfoque metafórico-analógico: numerosos componentes a diferentes escalas espacio-temporales}

Como fue estudiado, las "estructuras disipativas" poseen una inmensa cantidad de elementos o componentes accionando a diferentes escalas espacio-temporales (Cilliers, 1998). En la misma dirección, aunque en el ámbito de lo musical, actualmente pueden observarse obras que poseen dichos rasgos debido al uso de una notación estratificada o multidimensional (cabe destacar que Julio Estrada, compositor e investigador mexicano, fue uno de los principales artífices y constructores de esta clase de

de "complejidad" (o de "sistemas complejos") comprende casi inexorablemente al "caos", la "inestabilidad" (o no equilibrio) y la "no-linealidad" operando en simultáneo. Es decir, para que un sistema sea considerado como complejo, desde la mirada de este autor, parece necesario que haya caos, no equilibrio y no-linealidad (Prigogine y Stengers, 1990; Prigogine, 1997). Sin embargo, estos tres términos no son sinónimos y, dependiendo del enfoque o el área científica de la que se hable, a veces se pueden incluir -o no- dentro del ámbito de los sistemas complejos: por ejemplo, para Paul Cilliers (1998) el caos no se corresponde con la noción de complejidad. Por consiguiente, hay sistemas o modelos no-lineales con soluciones periódicas (un caso típico es el modelo "presa-predador" de Lotka-Volterra; o sea, en este caso no hay caos). Lo mismo sucede con el no equilibrio: no implica sí o sí la necesidad de caos (en el "equilibrio estable", el sistema se perturba, luego atraviesa cierta fase de no equilibrio, no obstante, pasado un tiempo, el sistema tenderá hacia el estado de equilibrio -ejemplo, el péndulo con fricción-; en el "equilibrio inestable", el sistema se encuentra en equilibrio, aunque, si se perturba, este pasa a otro equilibrio; si se repite esta operación en lo sucesivo, el sistema siempre pasará de un equilibrio a otro y por ello habrá inestabilidad -ejemplo, el péndulo sin fricción-). Ahora bien, con el "caos" debemos ser cuidadosos ya que no todos los especialistas van a coincidir en su definición. En tal sentido, Edward Lorenz (1995) apuntaba que dicho término ha cobrado diversos y variados significados técnicos que, sin embargo, están relacionados entre sí. Así pues, para Prigogine (según la interpretación de Lorenz) el caos se vincula al entendimiento de cómo es que algunos sistemas desorganizados adquieren organización. Otro tanto lo tenemos con Norbert Wiener quien a veces habla de caos, o varios tipos de caos, cuando se refiere a sistemas multitudinarios de moléculas con movimientos aleatorios (como una nube de gas). No contento con esto, el mismo Lorenz diferenciará el "caos limitado" del "caos completo". Pero va más allá, y nos dice que la noción de "equilibrio inestable" muchas veces se asocia con uno de los rasgos principales del caos, a saber: la "sensibilidad en las condiciones iniciales" (esto porque ambos involucran la amplificación de pequeñas diferencias en los inicios). Entonces, el caos limitado es aquel que posee (en sus ecuaciones) algunas soluciones aperiódicas, incluso cuando la mayoría de las soluciones puedan ser periódicas. Por el contrario, en el caos completo la probabilidad de encontrar comportamientos o soluciones periódicas es nula. De todos modos, una de las definiciones más aceptadas respecto al caos es: sistemas deterministas (no hay aleatoriedad en sus parámetros) de largo término con un movimiento aperiódico (debido a la no-linealidad) en donde la sensibilidad en las condiciones iniciales juega un rol crucial (Strogatz, 1994). En conclusión, no siempre resultará posible asociar a la no-linealidad y el no equilibrio con el caos. Asimismo, cabe destacar que nuestra metáfora-analogía no contempla una similitud o semejanza con el caos. 
notación; ver la serie de los yuunohui -1983 a 1991-para instrumentos de cuerda). Explicamos más detalladamente:

» Los muchos componentes se refieren a la cantidad de parámetros musicales en juego (técnicas instrumentales). Esto quiere decir que en una obra existen numerosas técnicas instrumentales (que generan timbres y sonoridades particulares) conviviendo de manera casi simultánea. Por ejemplo, si se trata de una obra para flauta, significa que podrían tenerse en cuenta los siguientes parámetros o técnicas instrumentales: ritmos con la boca (independientes de las manos), movimiento de la embocadura, fonemas, porcentajes de aire y sonido, intensidades, ritmo con ambas manos (alturas) y la voz.

» Las escalas aluden al nivel de resolución o refinamiento con que se desarrolla a cada uno de los componentes. Asimismo, en este trabajo se establecieron tres niveles o escalas: macro (implica a la totalidad de la obra); media (representa un refinamiento estándar; lo que generalmente se ha observado en la tradición); micro (propone mayor elaboración y detalle en cada uno de los parámetros o componentes). Por ello, las alturas podrían trabajarse del siguiente modo: nivel medio sería el temperamento igualitario mientras que la microtonalidad tiene que ver con el nivel micro. Así pues, cualquier parámetro es susceptible de ser trabajado con diferentes grados de minuciosidad.

Para entender mejor lo que explicamos arriba tomemos como punto de partida a Phowa (2018) para flauta bajo del autor de este texto. Los diferentes componentes y escalas quedarían organizados de la siguiente manera:

\begin{tabular}{llll}
\hline & Escala macro & Escala media & Escala micro \\
\hline A. Ritmo con la boca & $\begin{array}{l}\text { Es la totalidad de la } \\
\text { obra. }\end{array}$ & $\begin{array}{l}\text { Ritmos con la boca } \\
\text { independientes de lo } \\
\text { que realizan ambas } \\
\text { manos. }\end{array}$ & \\
\hline $\begin{array}{l}\text { B. Movimiento de la } \\
\text { embocadura }\end{array}$ & $\begin{array}{l}\text { Es la totalidad de la } \\
\text { obra. }\end{array}$ & Posición ordinaria. & Refina o amplía: \\
& & & $\begin{array}{l}\text { - Movimientos hacia } \\
\text { arriba y hacia abajo. } \\
\text { - Movimientos hacia } \\
\text { arriba y hacia abajo } \\
\text { muy irregulares } \\
\text { introduciendo } \\
\text { frullatis accidentales. }\end{array}$ \\
& & & \\
& Es la totalidad de la & Utilización de fonemas \\
que modifican el & \\
& sonido. & \\
\hline C. Fonemas & & & \\
& & &
\end{tabular}




\begin{tabular}{llll}
\hline $\begin{array}{l}\text { D. Porcentajes de aire } \\
\text { y sonido }\end{array}$ & $\begin{array}{l}\text { Es la totalidad de la } \\
\text { obra. }\end{array}$ & $\begin{array}{l}\text { Uso del tono puro } \\
\text { convencional. }\end{array}$ & $\begin{array}{l}\text { Refina o amplía: } \\
\text { - Mezcla de aire y } \\
\text { sonido. }\end{array}$ \\
\hline E. Intensidades & $\begin{array}{l}\text { Es la totalidad de la } \\
\text { obra. }\end{array}$ & $\begin{array}{l}\text { Desde un } p p p \text { hasta un } \\
\text { fff (lo que se observa } \\
\text { en la tradición). }\end{array}$ & $\begin{array}{l}\text { Refina o amplía: } \\
\text { F. Alturas }\end{array}$ \\
\hline G. Loz la totalidad de la & Temperamento igual. & \\
\hline obra. & $\begin{array}{lll}\text { Es la totalidad de la } \\
\text { obra. }\end{array}$ & $\begin{array}{l}\text { Uso de la voz } \\
\text { (glissandi). }\end{array}$ \\
\hline
\end{tabular}

Y así se plasma en el plano de la notación musical:

A: Ritmo con la boca

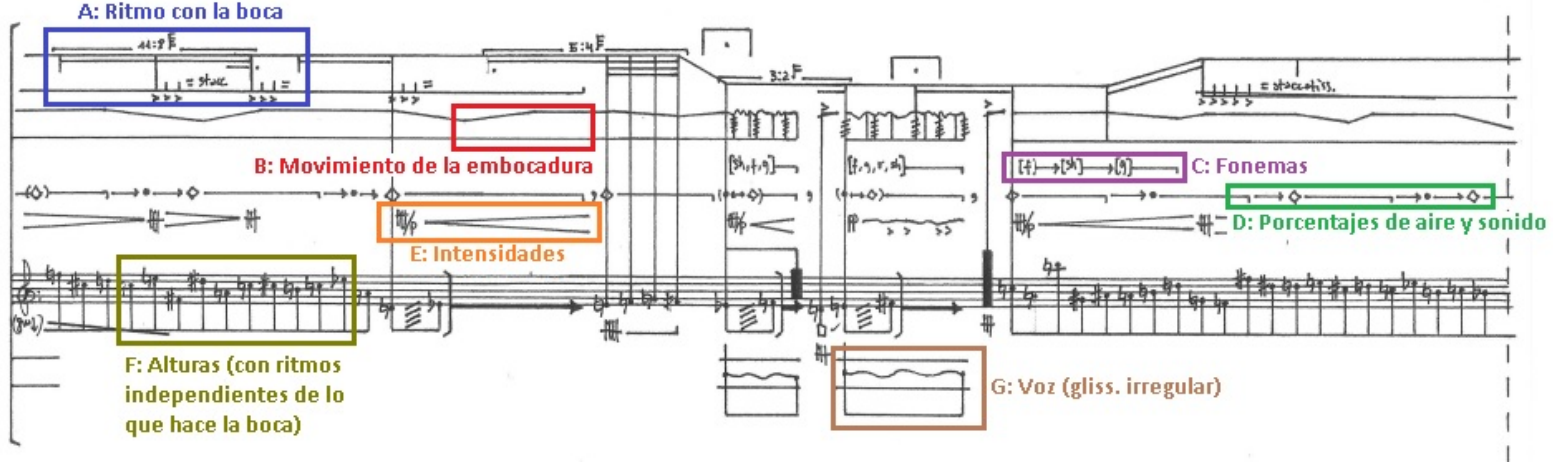

Imagen 1. Fragmento de Phowa (2018) de Pablo Araya para flauta bajo sola que muestra los diferentes componentes a distintas escalas interactuando entre sí.

4.2. Segundo enfoque metafórico-analógico: los componentes y escalas espacio-temporales con un comportamiento no-lineal

Lo mostrado en la sección anterior pone en evidencia la conformación estructural de obras que utilizan una notación estratificada. Ciertamente, esta clase de notación permite la construcción de objetos musicales con una complejidad tímbrico-textural considerable (es importante remarcar que esta no es la única forma de lograr tal cosa). No obstante, lo que resta por entender, es el "comportamiento dinámico" que dicha estructura de múltiples componentes y escalas contempla, o sea, saber cómo es que se "mueve" y "evoluciona" esa estructura en el tiempo. En tal sentido, puede decirse que esta clase de objetos musicales se vincula con los conceptos de "inestabilidad" y "turbulencia" (algo presente en las "estructuras disipativas" desarrolladas por Prigogine). Asimismo, ¿qué es lo que genera la inestabilidad y la turbulencia? Pues bien, antes de responder a dicho interrogante hagamos un breve repaso de los conceptos científicos que acabamos de nombrar y luego veamos cómo se materializan en el ámbito de lo musical: 
» Inestabilidad dinámica: cuando un sistema es perturbado constantemente, este puede alejarse del equilibrio, o, en su defecto, este puede atravesar estados de no equilibrio. Es decir, si la órbita en la que yace el sistema es perturbada lo suficientemente como para que el sistema se aleje de ella, pues entonces se entra en una zona de no equilibrio que, bajo ciertas condiciones, genera inestabilidad, turbulencia o caos (Von Bertalanffy, 1972; Briggs y Peat, 1990; Alligood, Sauer y Yorke, 1996).

" Turbulencia: lo que llamamos "turbulencia" se estudia en "mecánica de fluidos" (esto incluye el flujo del agua en un río, las nubes en el cielo, etc.). Básicamente, existen dos tipos de movimientos en los fluidos: el "laminar" y el "turbulento". El primero es un movimiento lento en donde las partículas del fluido se comportan como láminas o capas sin interferencias entre sí. Por el contrario, en lo que denominamos turbulencia, el movimiento del fluido es rápido y las trayectorias de las partículas se perturban e interfieren constantemente generando mucha inestabilidad. Esto generará estructuras cada vez más complejas e irregulares con cambios continuos, y, a su vez, también se observará un grado de libertad inusitado en el movimiento del fluido. Asimismo, desde un punto de vista un tanto más técnico lo turbulento implica, entre otras cosas, a la nolinealidad, la difusividad (capacidad de mezcla), la multiplicidad de escalas espacio-temporales, la disipación de energía, etc. (Uruba, 2012; Kessler, 2016).

» No-linealidad: este concepto muy usado en la física y la matemática de hoy, significa que los componentes de un sistema se encuentran estrechamente vinculados entre sí, lo que genera interferencias y complementaciones de diversa índole. Así, pequeñas causas pueden tener una influencia enorme sobre los otros componentes (y viceversa). Por lo tanto, un comportamiento no-lineal impide descomponer al sistema en partes para luego recombinarlas. Y también, esa vieja leyenda de lo lineal que dice que "el todo no es más que la suma de sus partes", es inaplicable para la ciencia de lo no-lineal: todos los componentes y escalas del sistema están, por más que cueste entender cómo, indisolublemente entrelazados. En conclusión, lo no-lineal implica que, si un parámetro, variable o componente es perturbado, pues entonces este influirá sobre todos los otros componentes que integren al sistema, aunque no de manera proporcional (Strogatz, 1994).

Ahora que ya tenemos una idea más acabada de los conceptos de inestabilidad dinámica, turbulencia y no-linealidad, observemos cómo se dan en el plano musical (cuestión que también permitirá responder a la pregunta que se realizó más arriba). Para ello tengamos en cuenta los siguientes razonamientos: parámetro o componente (con distintas escalas espacio-temporales) funcionando 
de manera no sincronizada o desfasada. Por ende, si cada elemento o técnica instrumental posee duraciones que no están sincronizadas en relación a las demás (y lo mismo sucede con las escalas), y que siempre se interfieren o complementan entre sí, lo que se obtiene es, en términos metafórico-analógicos, una gran inestabilidad dinámica: o sea, la obra funciona en condiciones de no-equilibrio y, en consecuencia, lo que se observa es la evolución de configuraciones o estructuras musicales aperiódicas o irregulares debido a la "perturbación" u oscilación constante de cada parámetro (también se percibe una gran riqueza tímbrica a partir de la "mixtura" de los componentes o parámetros) - (Araya, 2019b).

» En relación con la no-linealidad: si un parámetro es movido o perturbado (por ejemplo, el movimiento de la embocadura), este repercutirá e incidirá sobre los otros parámetros: las intensidades se alterarán, la altura también, y así podríamos seguir enumerando otras posibilidades (todo dependerá de la cantidad de elementos o técnicas que estén en juego en algún momento particular de la obra). Ahora bien, todo esto se logra codificar en una notación de tipo multidimensional (Araya, 2019a). Sin embargo, hay que tener en cuenta que esta clase de notación es deficiente para expresar (verdaderamente) a la nolinealidad: todos los parámetros aparecen distribuidos de forma proporcional y equitativa en la partitura (parece una representación lineal más que nolineal). De cualquier forma, en la realidad físico acústica de la obra, y a través del trabajo interpretativo del instrumentista, lo que habrá es no-linealidad. En síntesis, el modelo (notación) es deficiente (no está pensado en términos matemáticos, sino musicales), pero en el plano físico-acústico e interpretativo encontraremos que la no-linealidad es ineludible (Araya, 2019b).

Finalmente, y para clarificar las ideas recién expuestas, obsérvese el siguiente ejemplo:

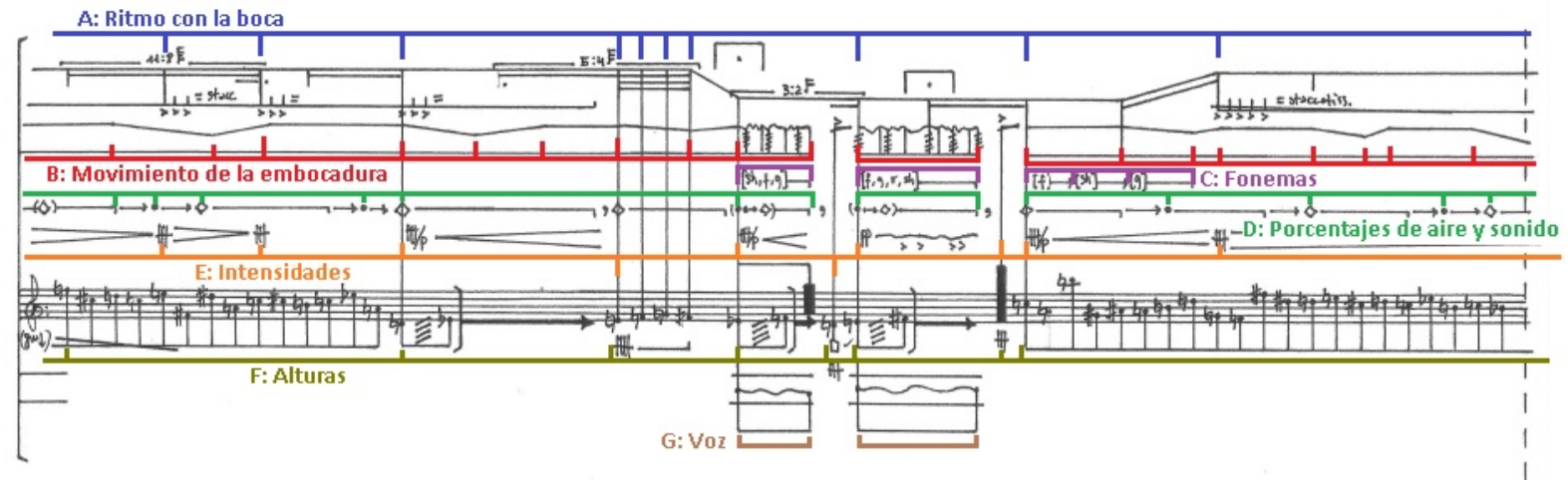

Imagen 2. Fragmento de Phowa (2018) de Pablo Araya para flauta baja sola, que muestra la simultaneidad de componentes y escalas espacio-temporales, cuestión que habilita a la no-linealidad, el no equilibrio y la turbulencia. 
4.3. Breves consideraciones respecto a los enfoques metafóricoanalógicos trabajados

En definitiva, y teniendo como referencia a las "estructuras disipativas" del Nobel en química, consideramos que nuestra metáfora-analogía resulta plausible. Es decir, existen correspondencias estructurales (las que ya expusimos en las secciones previas) entre el "dominio-fuente" (estructuras disipativas) y el "dominio-objetivo" (obra musical). Así pues, la cantidad elevada de componentes o elementos, lo multiescala (a nivel espacial y temporal), la idea de inestabilidad o no equilibrio, la turbulencia y la nolinealidad, que son atributos característicos de las "estructuras disipativas", se encuentran presentes, metafórica y analógicamente, en la obra que se tomó para el análisis (Phowa, para flauta baja). Solamente queda excluido el caos, es decir, la definición o concepción más técnica de los matemáticos (Strogatz, 1994) no fue tenida en cuenta; no se considera debido a que en la obra no se observa ninguna sensibilidad en las condiciones iniciales, y tampoco esta composición puede entenderse como un sistema de largo término; en última instancia, solo el movimiento aperiódico podría llegar a pensarse como una alternativa posible. De todas maneras, y con el fin de observar mejor cómo se plasma esta idea de Prigogine en lo creativomusical, véase el esquema gráfico a continuación (tomar como referencia a la imagen 2):

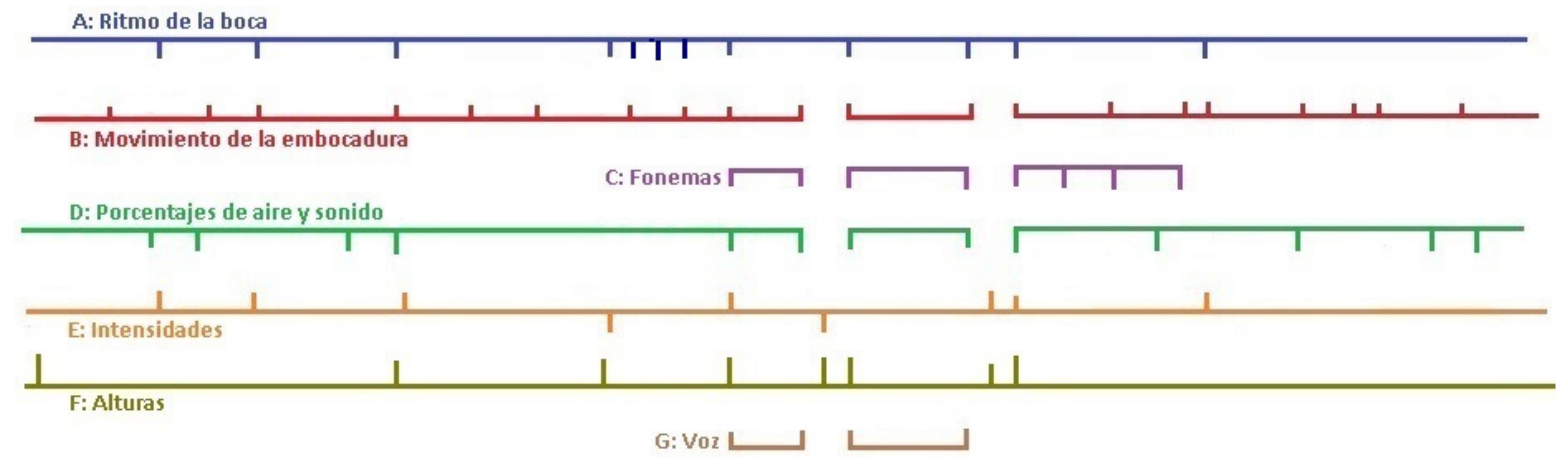

Imagen 3. Esquema gráfico que expone la convivencia simultánea (no-linealidad), aunque no sincronizada, de los diversos componentes o técnicas instrumentales, lo que genera inestabilidad dinámica y turbulencia.

\section{Conclusiones}

Según nuestro entendimiento, hay tres factores importantes en lo relativo a la temática abordada en este trabajo: 
»El diálogo entre las dos culturas propuesto por Prigogine no siempre puede llevarse a cabo de manera directa. En cambio, lo que aquí proponemos es un diálogo en donde lo artístico-musical y lo científico se ayudan y complementan en la resolución de problemas e inquietudes propias (correspondientes a cada campo).

» La metáfora y la analogía son los mecanismos que habilitan a que esta ayuda mutua entre música y ciencia (o a la inversa) cobre una existencia concreta.

» La metáfora-analogía con la noción de "estructuras disipativas" que aquí propusimos, aunque por momentos pueda parecer forzada y sin una suficiencia y contundencia completas en su formulación, en realidad está mostrando que dentro del ámbito de la "música contemporánea" existen obras que no pueden caracterizarse ni entenderse mediante términos y conceptos de la tradición musical anterior a la segunda mitad del siglo XX (por ejemplo, armonía, motivo, frase, etc.).

En conclusión y atendiendo a lo mencionado arriba, podría decirse que en la actualidad se observan nuevos mecanismos o resortes cognitivos al momento de estructurar e imaginar la música, especialmente aquella que incorpora los ruidos y otras experiencias tímbricas complejas. 


\section{Bibliografía}

» Alligood, K. T., Sauer, T. D. y Yorke, J. A. (1996). CHAOS: An Introduction to Dynamical Systems. New York, EEUU: Springer-Verlag.

» Amster, P. (2004). La matemática como una de las bellas artes. Buenos Aires: Siglo XXI Editores.

" Araya, P. (2019a). Objetos sonoros de múltiples componentes y escalas con un comportamiento no-lineal. Cuadernos de Análisis y Debate sobre Músicas Latinoamericanas Contemporáneas, 2(2), 145-162.

" Araya, P. (2019b). Una correspondencia metafórico-analógica conceptual entre ciencia y música: la noción de sistemas complejos aplicada al análisis de obras solistas y de cámara de Julio Estrada, Luciano Azzigotti, Samuel Cedillo y Raúl Dávila (tesis de doctorado en Artes -Música-). Universidad Nacional de Córdoba, Córdoba, Argentina.

» Beran, J. (2004). Interdisciplinary Statistics: Statistics in Musicology. Boca Raton, London, New York, Washington DC: Chapman \& Hall/CRC Press Company.

» Bohm, D. (2002). La totalidad y el orden implicado. Barcelona: Kairós (4ta edición).

» Briggs, J. y Peat, D. (1990). Espejo y reflejo: del caos al orden. Barcelona: Editorial Gedisa.

» Budón, O. (2000). Composing with Objects, Networks and Time Scales: An Interview with Horacio Vaggione. Computer Music Journal, 24(3), 9-22.

" Cilliers, P. (1998). Complexity and Postmodernism. London-New York: Routledge (Taylor \& Francis Group).

»Dutilh Novaes, C. (2013). Mathematical Reasoning and External Symbolic Systems. Logique \& Analyse, 221, 45-65.

» Fauvel, J., Flood, R. y Wilson, R. (Eds.). (2006). Music and Mathematics: From Pythagoras to Fractals. New York: Oxford University Press.

» Gelfert, A. (2016). How to do Science with Models: A Philosophical Primer. Switzerland: Springer.

» Goehr, L. (2017). All Art Constantly Aspires to the Condition of Music Except the Art of Music: Reviewing the Contest of the Sister Arts. En Kottman P. (Ed.), The Insistence of Art: Aesthetic Philosophy After Early Modernity (pp. 140-169). New York: Fordham University.

» Hacking, I. (2014). Why is there Philosophy of Mathematics at all? New York: Cambridge University Press.

" Hoppin, R. (1991). La música medieval. Madrid: Ediciones AKAL.

» Lakoff, G. (1993). The Contemporary Theory of Metaphor. En A. Ortony (Ed.), Metaphor and Thought (pp. 202-251). Cambridge: Cambridge University Press. 
» Lakoff, G. y Johnson, M. (1986). Metáforas de la vida cotidiana. Madrid: Editorial Cátedra Teorema.

» Lorenz, E. (1995). The Essence of Chaos. Seattle: Washington University Press.

» Macagno, F., Walton, D. y Tindale, C. (2016). Analogical Arguments: Inferential Structures and Defeasibility Conditions. Argumentation-Springer, 31(2), 221-243. doi: 10.1007/s10503-016-9406-6

» Nickerson, R. S. (2011). Mathematical Reasoning: Patterns, Problems, Conjectures, and Proofs. New York-London: Psychology Press Taylor \& Francis Group.

»Palau, G. (2003). Falacias y no-monotonía: ¿Hay una lógica informal? Epistemología e Historia de las Ciencias FFyH UNC, 9(9), 334-343.

»Pérez-Bernal, M. (2007). Metáfora frente a analogía: del pudín de pasas al fuego diabólico. Creatividad expresiva frente a creatividad cognitiva. Thémata Revista de Filosofía, 38, 201-211.

» Prigogine, I. (1997). ¿Tan sólo una ilusión? Una exploración del orden al caos. Barcelona: Tusquets Editores (colección metatemas).

»Prigogine, I. y Stengers, I. (1990). La Nueva Alianza - Metamorfosis de la Ciencia. Madrid: Alianza Editorial (2da edición).

»Strogatz, S. H. (1994). Nonlinear Dynamic and Chaos. EEUU-Canadá: Addison Wesley Publishing Company.

»Uruba, V. (2012). Turbulence Handbook for Experimental Fluid Mechanics Professionals. Skovlunde: Dantec Dynamics A/S.

»Von Bertalanffy, L. (1972). The History and Status of General Systems Theory. The Academy of Management Journal, 15(4), 407-426.

»Walton, D. (2006). Fundamentals of Critical Argumentation. New York: Cambridge University Press.

»Walton, D. y Hyra. C. (2018). Analogical Arguments in Persuasive and Deliberative Contexts. Informal Logic, 38(2), 213-261.

»Walton, D., Reed, C. y Macagno, F. (2008). Argumentation Schemes. New York: Cambridge University Press.

»Weisberg, M. (2013). Simulation and Similarity: Using Models to Understand the World. New York: Oxford University Press.

»Wilber, K. (Ed.). (2007). Cuestiones cuánticas. Barcelona: Editorial Kairós.

»Wigner, E. (2004 [1960]). La irrazonable eficacia de la matemática en las ciencias naturales. Communications in Pure and Applied Mathematics, 13(1), 1-12.

»Wollenberg, S. (2006). Music and Mathematics: An Overview. En Fauvel, J., Flood, R. y Wilson, R. (Eds.). Music and Mathematics, From Pythagoras to Fractals (pp. 1-9). New York: Oxford University Press.

» Xenakis, I. (1971). Formalized Music. Bloomington-London: Indiana University Press. 


\section{Otras fuentes consultadas}

》Amster, P. (2019, 12 de septiembre). Para las seis cuerdas. Medium.

Recuperado de https://medium.com/@amster.pablo/para-las-seis-

supercuerdas-6aedaa213ca

» Bartha, P. (2013). Analogy and Analogical Reasoning. The Stanford

Encyclopedia of Philosophy. California: enciclopedia. Recuperado de https:// plato.stanford.edu/archives/spr2019/entries/reasoning-analogy/

» Kessler, M. (2016, 18 de octubre). Flujo turbulento. ESSS. Recuperado de https://www.esss.co/es/blog/flujo-turbulento/

»Visokolskis, Sandra (2006, 7 y 8 de septiembre). Metáfora, ícono y abducción en C.S. Peirce. II ${ }^{\mathrm{a}}$ Jornadas Peirce en Argentina. Recuperado de http://www. unav.es/gep/IIPeirceArgentinaVisokolskis.html

\section{Partituras}

Araya, P. (2018). Phowa. Córdoba.

\section{Biografía/Biografia/Biography}

\section{Pablo Federico Araya}

Licenciado en Composición Musical en la Universidad Nacional de Córdoba. Recientemente obtuvo el Doctorado en Artes en la misma universidad bajo la dirección de Andrés Barrea (UNC-FAMAF) y Julio Estrada (UNAM-México), con la tesis "Una correspondencia metafórico-analógico conceptual entre ciencia y música: la noción de sistemas complejos aplicada al análisis de obras solistas y de cámara de Julio Estrada, Luciano Azzigotti, Samuel Cedillo y Raúl Dávila". Ha sido becario del CONICET (Consejo Nacional de Investigaciones Científicas y Técnicas), del CELFI (Centro de Estudios Latinoamericanos de Formación Interdisciplinaria), de la Secretaría de Posgrado de la Facultad de Artes de la UNC (2014-2019) y del Fondo Nacional de las Artes. Ha tomado numerosos cursos de composición con Julio Estrada, Pierluigi Billone, Georges Aperghis, C.S. Mahnkopf, Dai Fujikura, Brian Ferneyhuoguh, Juan Carlos Tolosa, Mark André, Marcos Franciosi, Gabriel Valverde, etc. Trabajó con diversos solistas y ensambles: David Núñez, Beatrix Wagner, Eduardo Spinelli, Émilie Girard-Charest, Dúo de flautas MEI, Cuarteto de Cuerdas de la UNTREF, Ensamble Reflexion K (Alemania). 